\title{
8th EASD/JDRF OXFORD WORKSHOP
}

\section{"Prediction and Early Detection of Diabetic Complications: Common Pathways and Unique Needs"}

\section{Keble College, Oxford \\ August 1-4, 2003}

\section{Meeting format and purpose}

The European Association for the Study of Diabetes and the Juvenile Diabetes Research Foundation jointly convene a once yearly Oxford Workshop. This joint workshop attempts to focus a small group of researchers on a specific area of relevance to Type 1 diabetes and its complications. The meeting participants are given the task of identifying emerging opportunities and key areas of need in the research areas addressed, so that the two agencies can use this advice to better focus their research funding programmes and activities. These meetings are informal in nature, with a strong emphasis on audience discussion of topics presented. Previous workshops on diabetes complications include the second Oxford Workshop, on Cardiovascular Disease and Diabetes, the third Oxford Workshop on Diabetic Retinopathy and the sixth Oxford Workshop on Molecular and Genetic Aspects of Vascular Complications of Diabetes. The goal of the sixth Workshop was to survey progress concerning insights into the mechanisms of vascular complications of diabetes.

The eighth EASD/JDRF Oxford Workshop, 'Prediction and Early Detection of Diabetic Complications: Common Pathways and Unique Needs', addressed a number of fields of relevance to current research on diabetes complications. The emphasis of this workshop was, in particular, on the link between basic research and diabetic complications, bringing together experts from various fields of expertise, such as vascular biology, array technologies, bioinformatics, and experimental and clinical diabetology.

This was a thought-provoking meeting, comprised of brief presentations by senior scientists along with junior fellows from their research laboratories, followed by substantial discussion and informative, humorous and sometimes contentious questions from the group.

The recommendations overview that accompanies this summary attempts to capture the tenor of the meeting and in addition, several key points are laid out, indicating areas of need that funding agencies can consider as potential opportunities to pursue.
Following this is a brief summary of speaker presentations and the discussions that followed.

\section{Workshop recommendations: overview}

Therapeutics for the treatment of Type 1 diabetes and its complications are currently still in the early stages of clinical development. However, some critical basic research has recently begun to offer the promise of successful interventions being made available to the patient in the not-too-distant future. This was the underlying theme of the eighth EASD/JDRF Oxford Workshop, 'Prediction and Early Detection of Diabetic Complications: Common Pathways and Unique Needs'.

A key recommendation from the participants at this workshop was that funding agencies should continue to promote interdisciplinary interactions. Valuable lessons from other disciplines can be learned and brought into the diabetes research arena, including:

- Angiogenesis: Vascular biology is a maturing field, and the understanding of the complexity of vessel formation may become beneficial for such diverse areas as that of diabetic retinopathy and wound healing.

- Developmental biology: Expertise from this area can complement existing diabetes-related research and may be of particular value in the nascent field of stem cell biology.

- Metabolism and biochemistry: Research on obesity is beginning to have an influence on the diabetes field, which can capitalize on this by recruiting new researchers into diabetes research. In addition, new techniques can be brought to bear to study existing metabolic pathways. A possible way to generate renewed interest in these fields is with the development of 'bioanalytical centres'.

- Genomics: This area of research is gradually being brought into the diabetes arena and has great potential value in the development of novel diagnostics and surrogate markers for predicting the progression of diabetic complications. 
- Proteomics: Research efforts such as the Human Plasma Proteome hold the promise of a revolution in disease diagnosis and therapeutic monitoring. Although major challenges in the proteomics and related fields still lie ahead, this field has the potential to rapidly produce novel indicators of disease progression.

A suggestion was made that funding agencies should continue to aggressively pursue these new opportunities and where possible, should begin to pro-actively export diabetes-related research into other fields of inquiry.

\section{Clinical and translational research}

Another key area of discussion at the workshop was that of translational research. While the utility of basic research is still key to our understanding of diabetic complications, translational, "bench-to-bedside" research is the ultimate goal of the funding agencies. Some concerns were raised at the workshop regarding the difficulty of predicting what are good therapeutic candidates to pursue. Suggestions were made that the funding agencies should exercise some caution when choosing which individual candidate therapeutics to go after.

A recurrent theme at this and at previous Oxford workshops is that clinical trial infrastructure in the area of complications research is still incomplete and could benefit from funding agency support. Funding agencies should continue to work towards fostering relations with regulatory agencies such as the Food and Drug Administration and with equivalent European counterparts. A good understanding of the regulatory hurdles encountered when applying for Investigational New Drug (IND) authorizations is a key area where funding agencies can facilitate translational research. Funding agencies might consider supporting researchers to successful Phase I or Phase II clinical trials and should continue to foster relationships with the biotechnology industry with this goal in mind.

Valuable lessons can be learned from studies evaluating differences between Type 1 and Type 2 diabetes complications and there is currently a paucity of epidemiological data addressing this issue. Although the mechanisms underlying hyperglycaemic damage are the same, there may be underlying genetic factors that either worsen or protect from progression to certain complications. Where existing and future clinical or epidemiological studies are concerned, the careful storage of sample collections in centralized repositories (such as the NIDDK sample repository) should be considered whenever appropriate.

\section{Summary of Workshop recommendations}

\section{Implications of the DCCT/EDIC study}

The DCCT/EDIC study clearly demonstrated that intensive treatment to haemoglobin $\mathrm{A}_{1} \mathrm{c}\left(\mathrm{HbA}_{1} \mathrm{c}\right)$ of 7.2 percent leads to marked reduction in the cumulative incidence of diabetes-related complications. Following the DCCT, investigators predicted that the divergence in cumulative incidence of complications between intensive and conventional treatment groups would diminish, however, this did not occur. Furthermore, during the follow-up EDIC study, the initial intensive and conventional groups were treated almost identically, with their $\mathrm{HbA}_{1} \mathrm{c}$ levels converging, yet the originally assigned intensive cohort continued to show persistently decreased complications. This phenomenon has been referred to as "metabolic memory" or "imprinting" and has been proposed to explain the persistent effects of glycaemia on long-term diabetic complications.

Hyperglycemia then, leads to physiological changes that ultimately determine the risk of complications. Tissue damage from any level of hyperglycaemic exposure outlasts the period of exposure and blunts subsequent responses to intensive treatment. Recent work by Michael Brownlee's group has suggested that there are four key metabolic pathways activated by a single, hyperglycaemia-induced process: the formation of highly reactive superoxide. The over-production of superoxide inhibits the enzyme GAPDH (glyceraldehydes 3-phosphate dehydrogenase) that redirects metabolites from glycolysis into the four metabolic pathways. These metabolic pathways include the activation of protein kinase $\mathrm{C}$ (PKC), increased hexosamine pathway flux, increased advanced glycation end-product (AGE) formation and activation of aldose reductase, all of which lead to chronic hyperglycaemic damage. In addition, the cumulative chronic effects of hyperglycaemia may be due to the production of AGEs, which have previously been associated with the severity of complications.

Since the DCCT and EDIC studies have demonstrated that risk reduction is possible, overall risk reduction as a clinical endpoint may prove critical in the design of future clinical trials and should not be overlooked. Risk reduction is desirable as a short-term goal for addressing diabetic complications, with the obvious long-term strategy of complete prevention.

Clearly then, there are now a number of potential points of therapeutic intervention at which to block the progression to microvascular complications, and chronic tissue and organ damage. These potential therapeutic interventions were discussed at length during the workshop.

A summary of the findings of the DCCT/EDIC study can be found at:

http://www.niddk.nih.gov/fund/other/ metabolic-imprinting/Overal-Sympoiessions.pdf

\section{Therapeutic strategies to prevent or reverse microvascular complications}

There are a number of potential strategies to pursue to prevent or possibly reverse damage caused by hyper- 
glycaemia. Typically, the most effective strategies would be aimed at the vasculature, which appears to be the first organ system to undergo hyperglycaemiainduced damage. Some current therapeutic candidates that were discussed include:

- Antioxidant combinations: Antioxidants aimed at blocking oxidative stress pathways causing intracellular damage can be combined to increase their efficacy.

- Benfotiamine: A synthetic thiamine derivative that activates the enzyme transketolase to redirect glycolytic metabolites from vascular damaging pathways.

- Inhibition of AGE formation and RAGE activation: Small molecule inhibitors of the receptor for AGEs (RAGE) and agents that prevent AGE accumulation such as cross-link breakers to prevent the activation of this receptor are now being developed for therapeutic use.

- Aldose reductase inhibitors: Although previously these have often proved to be too toxic or sometimes inefficient to use in humans, less toxic aldose reductase inhibitors to block activation of the polyol pathway are currently under development in the pharmaceutical industry.

- PKC inhibitors: Eli Lilly's PKC inhibitor, ruboxystaurine is now in Phase III clinical trials and has proved effective in blocking certain aspects of complications in several pilot studies.

- PARP inhibitors: Perhaps one of the most promising of therapeutic strategies is to block the enzyme poly (ADP-ribose) polymerase (PARP) which is activated by the DNA strand breakage caused by superoxide overproduction. This enzyme in turn inhibits the activity of GAPDH. In laboratory experiments, PARP inhibitors have been shown to prevent hyperglycaemia-induced activation of all of the major metabolic pathways.

- Angiogenesis inhibitors: In addition to these strategies to prevent microvascular damage, anti-angiogenesis therapies may lead to promising treatments in special areas of complications in which new vessel formation can cause tissue damage.

A further recommendation from the workshop was that diabetes researchers could learn lessons from the cancer and AIDS fields, where combined therapeutic strategies have been proven to work. Combination therapy is the main research area addressed by the JDRF Centre for the Study of Complications in Diabetes at the University of Michigan with a focus on experimental diabetic neuropathy.

Since there is now quite a number of therapeutics in the pipeline, there is a growing need for in vivo, pre-clinical research with novel therapeutics and with combined therapeutic use in good animal models of diabetic complications to assess both their safety and efficacy prior to clinical trials in humans. With this in mind, novel animal models of complications are now being developed by the US NIH through the National Institute of Diabetes and Digestive and Kidney Disease (NIDDK) animal models of diabetes complications consortium (AMDCC) (http://www.amdcc.org).

\section{What can new technologies offer to diabetes research?}

A number of new technologies and their potential for use in diabetes research were discussed at the workshop and there are several areas where these technologies can be exploited. These are discussed below:

- Genomics: Several genomics initiatives are now underway and genomics has great potential in the development of diagnostic biomarkers for future clinical studies.

- Proteomics: Several proteomics initiatives are currently in the pipeline. In the USA, a recent NIDDK programme announcement was made specifically for funding of collaborative projects on proteomics and metabolomics for Type 1 diabetes and its complications (http://www.niddk.nih.gov/fund/diabetesspecialfunds/proteomics/). In addition $\mathrm{NIH}$ funds are now available for small business grants for high throughput proteomics.

- Metabolomics: It has long been recognized that levels of blood metabolites such as lipids or byproducts of cellular reactions can indicate disease. What makes metabolomics new is the systematic attempt to tabulate and quantify all the small molecules within a sample to find new markers for disease or drug toxicity. An obvious use for metabolomics therefore, is in the search for new disease markers since thousands of metabolites present in a sample can be scanned, looking for ones that correlate either positively or negatively with disease, with the goal of identifying new prognostic indicators. Although few public databases exist, one that includes mass spectra for thousands of human metabolites is maintained by the National Institute of Standards and Technology (NIST) (http://webbook.nist.gov/).

- Informatics initiatives: There is a need for wider dissemination of information on transgenic animals available to researchers. This is especially true where specific transgenic animals have been generated which affect specific metabolic pathways. Where possible, the funding agencies can facilitate wider access to transgenic animals through specific initiatives such as the Animal Models of Diabetes Complications Consortium (AMDCC) (http://www.amdcc.org). 


\section{Meeting participants:}

Keynote lecturers: Saul Genuth (Case Western Reserve University, Cleveland USA), Jennifer LeCouter (Genentech Inc., South San Francisco, USA), Christer Betzholtz (University of Goteburg, Sweden), Gavin Thurston (Regeneron Inc., New York, USA), Michael Brownlee, (Albert Einstein College of Medicine, NY, USA).

Senior scientists and Fellows: Hans Peter Hammes (Chair) and Angelika Bierhaus (University of Heidelberg, Germany) Mark Cooper (Co-Chair) and Josephine Forbes (Baker Heart Research Institute, Australia), Peter James (Lund University, Sweden), Steven Rich and Michèle Sale (Wake Forest University, USA), Anand Swaroop and Monica Buraczynska (University of Michigan, USA), Maria Grant and Aqeela Afzal (University of Florida, USA), Klaus Preissner and Heidi Trusheim (University of Giessen, Germany), Roy Bicknell (Oxford University, UK), Csaba Szabó and Jon Mabley (Inotek Corp, Beverly MA, USA), Eva Feldman and Andrea Vincent (University of Michigan, USA), Philip Low and Haruyasu Iida (Mayo Foundation, MN USA), Ann Marie Schmidt and Ravi Ramasamy (Columbia University, NY, USA), Andrew Boulton and Cuong Dang (University of Manchester, UK), Erwin Bottinger and Katalin Susztak (Albert Einstein College of Medicine, NY, USA), Stuart Shankland and Raghu Durvasula (University of Washington, WA, USA), Hermann Haller and Matthias Meier (Medizinische Hochschule, Hanover, Germany), Kumar Sharma and Satish Rao (Thomas Jefferson University, PA, USA), Giancarlo Viberti and Giorgia Setti (King's College London, UK), Peter Nawroth and Peter Reismann (University of Heidelberg, Germany) and Renee Kinman (Case Western Reserve, Cleveland, USA).

\section{Meeting Overview}

This workshop brings together basic researchers in different areas of study together with researchers in the diabetes field. The invited speakers tackled several issues related to diabetic microvascular complications and attempted to outline common pathophysiological mechanisms and potential therapeutic strategies. In addition, the speakers gave an overview of emerging technologies and therapeutic strategies that are beginning to be utilized in the laboratory and clinic. The talks focused on several interrelated themes and included a fresh look at hyperglycaemia and signalling, diabetic neu- ropathy and wound healing, angiogenesis and vascular biology, diabetic retinopathy and diabetic nephropathy, The talks were followed by a round table discussion on the final day of the meeting (summarized in part I).

\section{Hyperglycaemia \& signalling}

Saul Genuth gave a keynote lecture providing an overview of the findings from the DCCT/EDIC study on the effects of intensive treatment and glycaemic exposure on microvascular complications. The DCCT study recruited 1,441 participants in 1983, and was followed up by the EDIC study, which then reported its findings in 2002. The DCCT study originally consisted of an initial phase involving a randomized clinical trial of intensive versus conventional insulin therapy, followed by a secondary follow-up phase that examined long-term effects in order to determine the risk factors for developing complications influenced by treatment. A reduction in the cumulative incidences of diabetic retinopathy, nephropathy and neuropathy by up to 75 percent was gained by intensive treatment, which reduced haemoglobin $\mathrm{A}_{1 \mathrm{C}}\left(\mathrm{HbA}_{1} \mathrm{c}\right)$ levels to 7.2 percent, compared to conventional treatment with $\mathrm{HbA}_{1 \mathrm{c}}$ levels at 9.0 percent. This result demonstrated that reduction of glycaemic levels over an extended period eventually overcomes the influence of prior hyperglycaemia. The beneficial consequences of intensive treatment were not obvious until three to four years of DCCT follow-up, and the beneficial effects were further enhanced in those with a shorter duration of diabetes. Thus, the previous glycaemic history of a person with diabetes clearly influences the future course of the disease.

The DCCT findings also demonstrated that hyperglycaemia has an influence on the mechanisms of tissue damage. Intensive treatment has provided beneficial effects on retinopathy, nephropathy and neuropathy that have persisted for eight years, despite the near equalization of glycaemia during that time period. In addition in the EDIC cohort, a reduction in risk of atherosclerosis as measured by computed tomography (CT) and increases in carotid artery intimal medial thickness (IMT) and coronary artery calcification (CAC) were also observed, with the effects of conventional treatment showing significantly greater increases for these risk factors.

Other risk factors include the accumulation of advanced glycation endproducts (AGEs) that were measured in skin collagen. Towards the end of the DCCT study, these markers were lower in the intensive group than in the conventional group and correlated cross- 
sectionally with diabetic complications, independent of $\mathrm{HbA}_{1 \mathrm{c}}$ levels. The AGEs, in particular carboxymethyl-lysine (CML), were also predictive of the progression to retinopathy (examined four years after DCCT, during the EDIC study) and were again independent of $\mathrm{HbA}_{1} \mathrm{c}$ levels.

The overall conclusions suggested by DCCT/EDIC findings were (i) that the benefits of intensive treatment are due to a reduction in hyperglycaemia, (ii) tissue damage from any level of glycaemic exposure outlasts the period of exposure and blunts subsequent responses to intensive treatment and (iii) the long lasting effects may be due to the production of AGEs.

\section{Do different pathogenic mechanisms result from a single common pathway?}

Clinical studies such as the DCCT/EDIC study show a strong association between glycaemia and diabetic microvascular complications in both Type 1 and Type 2 diabetes. Michael Brownlee's talk "A Unified Mechanism for the Pathogenesis of Diabetic Complications" provided a detailed examination of the evidence for a single pathogenic mechanism that could explain the underlying features of hyperglycaemia-induced cellular damage.

Hyperglycaemia induces a series of pathological changes in capillaries, arteries, peripheral nerves and other tissues. Four main hypotheses for how hyperglycaemia causes diabetic complications have previously been generated. These hypotheses are: increased glucose flux through the aldose reductase pathway; increased advanced glycation end product (AGE) formation; activation of protein kinase $\mathrm{C}$ (PKC) isoforms; and increased flux through the hexosamine pathway. Until recently, there was no unifying hypothesis linking these mechanisms. However recent work suggests that activation of these four molecular pathways may result from the overproduction of mitochondrial superoxide. The importance of these pathways is supported by in vitro studies in which specific inhibitors of aldose reductase, PKC and AGE formation have been demonstrated to prevent hyperglycaemia induced changes in different organs. However, if one moves upstream, by normalizing mitochondrial superoxide production with specific inhibitors that target electron transport chain complex proteins, uncoupling proteins or manganese superoxide dismutase, it is possible to prevent glucose-induced sorbitol accumulation, activation of PKC and NF- $\kappa \mathrm{B}$ in cultured endothelial cells.

Hyperglycaemia-induced overproduction of superoxide by the mitochondrial electron transport chain has been shown to activate pathways of hyperglycaemic damage in aortic endothelial cells through inhibition of glyceraldehyde-3-phosphate dehydrogenase (GAPDH) activity. The inactivation of GAPDH diverts upstream glycolytic metabolites into the PKC, hexosamine and polyol pathways. This is supported by experiments where anti-sense oligonucleotides were used to inhibit GAPDH, recapitulating the effects of hyperglycaemia in cultured endothelial cells.

GAPDH is inhibited by the enzyme poly (ADP-ribose) polymerase (PARP), which is activated by DNA strand breaks produced by mitochondrial superoxide overproduction. In a talk by Csaba Szabó and Jon Mabley, the effects of PARP and GAPDH inhibition were discussed in the context of diabetes. Upon binding to DNA strand breaks, activated PARP cleaves $\mathrm{NAD}(+)$ into nicotinamide and ADP-ribose and polymerizes ADP-ribose onto nuclear acceptor proteins such as histones, transcription factors and PARP itself. Activation of PARP in response to oxidative stressmediated DNA single strand breaks promotes cellular dysfunction and necrotic-type cell death in a variety of pathophysiological conditions. The activation of PARP occurs in cultured endothelial cells incubated in high glucose concentrations, and is thought to be due to the generation of reactive oxygen and nitrogen species. In addition, PARP activation has been associated with the development of diabetic cardiomyopathy in animal models and has also been observed in human diabetic microvessels in the skin where it is seen early after diabetes onset and correlates with the severity of endothelial dysfunction.

The chronic long-term effects of diabetic complications may be due to the accumulation of advanced glycation endproducts (AGEs). Peter Nawroth and Peter Reismann discussed the role of AGE-related signalling in the context of the chronic long-term effects of hyperglycaemia. Receptors for AGEs (RAGE) serve a dual function, they perform the task of aiding elimination of AGE-modified proteins and in addition, these receptors play a role in cellular signalling. The signalling pathways induced by AGE receptor activation can lead to the generation of ROS, and may deplete intracellular antioxidant defence mechanisms and in addition, may activate the redox-sensitive transcription factor NF-кB. Activation of this transcription factor may then lead to the expression of a variety of pro-inflammatory markers that have previously been associated with the diabetic milieu. This AGEgenerated oxidative stress, coupled with transcription factor activation, could potentially explain the changes in the vascular endothelium leading to the prothrombotic state, vasoconstriction and impaired microcirculation. In addition, activation of the AGERAGE NF- $\kappa \mathrm{B}$ activation pathway has been demonstrated in the dorsal root ganglion (DRG) of diabetic wild-type but not RAGE-deficient animals, suggesting a further role for this pathway in the pathogenesis of neuropathy and possibly other complications.

The RAGE pathway has also been implicated in the pathogenesis of diabetic macrovascular complications. In a talk by Drs. Anne Marie Schmidt and Ravi Ra- 
masamy entitled: "Insights into Pathogenic Mechanisms in Diabetic Atherosclerosis and Cardiac Dysfunction", the role of RAGE was discussed in relation to macrovascular dysfunction. RAGE is a multiligand binding member of the immunoglobulin superfamily of proteins. Recent studies have revealed a number of signal transducing ligands of RAGE, which include: AGEs - such as carboxymethyl-lysine (CML) adducts of proteins/lipids that are highly prevalent in vivo; S100/calgranulins - cytokines that play a role in inflammation; amphoterins - heparin-binding proteins that play a role in neural development and inflammation, and amyloid $\beta$ peptide, which plays a role in Alzheimer's disease. Ligand activation of RAGE leads to up-regulation of the receptor and initiates a "vicious cycle' of sustained cellular perturbations, with amplified levels of RAGE on the cell surface making it a target for further ligand interactions that in turn propagates further cellular dysfunction. Dr. Schmidt's group tested the function of RAGE using a number of murine models. A common feature of diabetic vascular complications is enhanced vascular leakage and neointimal expansion. RAGE-deficient mice are viable, and after streptozotocin-induced diabetes, display a striking decrease in neointimal expansion in response to arterial injury. In addition, RAGE-mediated signalling via the JAK/STAT signal transduction pathway has been implicated in signalling in response to this injury. Work by Dr. Schmidt's group has further focused on the role of RAGE in the generation of atherosclerotic lesions. Using ApoE-deficient mice that are prone to accelerated atherosclerosis, Dr. Schmidt's group demonstrated that this enhanced atherosclerotic lesion could be blocked by the addition of a soluble form of RAGE (sRAGE). Furthermore, there was a dramatic stabilization of existing atherosclerotic lesions while at the same time, no changes in overall lipid profiles were observed, suggesting that these effects are conferred via RAGE signal amplification.

\section{Oxidative stress, signalling and diabetic neuropathy}

Although the precise pathogenesis of diabetic neuropathy is unknown, a number of putative pathophysiological mechanisms exist. Hyperglycaemia results in the induction of oxidative stress, polyol pathway overactivity, increased AGE formation, nerve hypox$\mathrm{ia} /$ ischaemia, deficiency of $\gamma$-linolenic acid, increased activation of the protein kinase $\mathrm{C} \beta$-isoform, and growth factor(s) deficiency. These pathways all converge in producing oxidative stress, which appears to be more severe at the DRG than at the nerve. Two talks on oxidative stress and diabetic neuropathy were given by Phillip Low and Haruyasu Iida and Eva Feldman and Andrea Vincent.

Dr. Phillip Low and Dr. Haruyasu Iida discussed the merits of using experimentally induced diabetic neuropathy. Their model of experimental diabetic neuropathy (EDN) closely mimics the human condition. The diabetic state in humans results in a primarily length-dependent sensory neuropathy. In rodents, EDN induced by streptozotocin is also a predominantly length-dependent sensory neuropathy. Beyond 6 months of diabetes, diabetic rats develop a number of pathological changes including mitochondrial swelling and vacuolar degeneration of dorsal root ganglion (DRG) neurons. These observations have led to the present hypothesis that a primary target of diabetic neural complications is the sensory neuron. There is electrophysiological and pathological evidence of targeting of nerve microvessels, Schwann cells and DRG neurons in human diabetic neuropathy. Phillip Low described work in his laboratory that has focussed on these same three targets in EDN. Reactive oxygen species (ROS) and especially ischaemia-associated ROS result in mitochondrial membrane depolarization and release of cytochrome c, which binds to the transcription factor Apaf-1 and thus activates caspase-9 and other downstream caspases especially caspase-3 (a principal effector in the apoptotic pathway in neurons and their support cells). The Low group evaluated oxidative injury as a function of duration of diabetes in the DRG of rats with EDN over a 12-month period. Deficits in nerve conduction were observed within one month and had progressed. This phenomenon is correlated with oxidative injury, which occurred at all timepoints throughout EDN, but was most prominent at 12 months. Caspase- 3 expression and TUNEL positivity are also increased in EDN and were seen at all timepoints. Furthermore, ROS causes neuronal DNA damage, especially to the mitochondria of DRG and Schwann cells, which demonstrate some mitochondrial DNA mutations. In addition, there is an overall reduction in the number of the largest DRG neurons in EDN, suggesting that activation of the apoptotic pathway may mediate some of the pathology of diabetic neuropathy. Taken together, these findings suggest that chronic hyperglycaemia results in a number of pathophysiological changes that appear to result in oxidative stress within DRG neurons. With increased duration of experimental diabetes, markers of oxidative injury are correlated with mitochondrial dysfunction, caspase 3 activity and TUNEL positivity, supporting the proposition that oxidative injury is responsible for the apoptosis seen in these neurons.

Eva Feldman and Andrea Vincent gave a complementary talk, which also discussed the role of oxidative stress and mitochondrial dysfunction in primary cultures of DRG neurons. Similarly, they reported the activation of caspase 9 followed by that of caspase 3 in response to high concentrations of glucose $(45 \mathrm{mM})$ and other parameters akin to the findings of the Low group. In these primary neuron cultures, certain antioxidants can block cell injury induced by high glucose concentrations. The antioxidants $\alpha$-lipoic acid 
and taurine can also block oxidative stress in the DRG of diabetic rodents and can prevent the onset of diabetic neuropathy. Furthermore, the addition of antioxidants after a rodent has developed diabetic neuropathy can slow progression of the disease and partially restore function. This has led to the speculation that these strategies can be utilized in research and treatment paradigms in man. Dr. Feldman went on to discuss a clinical study that her centre at the University of Michigan is currently conducting, utilizing a combination of antioxidants to intervene at different points along the oxidative stress pathway and potentially halt the progression of diabetic cardiac autonomic neuropathy.

\section{Wound healing}

Drs Andrew Boulton and Cuong Dang gave a talk entitled "new frontiers in diabetic ulceration". During the normal course of wound healing there are several characteristic phases, including inflammation, cellular proliferation and remodelling. Chronic non-healing wounds occur when the process is compromised, and ultimately chronic wounds may fail to heal due to decreased growth factor activity or increased protease activity or both. A number of different growth factors and cytokines play a role in wound healing and are important in all three wound healing phases. These factors promote chemotaxis and mitogenesis, stimulate angiogenesis and influence the synthesis and degradation of extracellular matrix proteins. In the diabetic setting, certain growth factors display altered expression profiles. For example, TGF- $\beta 1$ and IGF1 expression are perturbed in diabetic foot ulcers (DFU) and may contribute to the chronicity of retarded wound healing. In addition, matrix metalloproteinases (MMPs) 1, 2, 8 and 9 have been shown to be increased in chronic DFU compared to control nonDFU, whereas the expression of TIMP-2 is decreased two-fold in DFU. Such findings suggest that the increased proteolytic environment may also be contributory to the chronicity of DFUs. Additional factors that may play a role include nitric oxide, which is involved in cell-mediated cytotoxicity and endothelium-dependent vascular smooth muscle relaxation. A number of factors that enhance wound healing were also discussed and the emerging theme from this illuminating presentation was that wound healing rates can be drastically improved when patients are given proper standards of care, which include the control of infection by frequent wound debridement and vascular reconstruction for patients with severely compromised peripheral circulation. However, most critically, proper offloading of wounds appeared to significantly improve the rate of wound healing in DFU and could explain why several commercial treatments for diabetic wounds failed in clinical trials. Persons with DFU may not adhere to standard pressure-offloading regimens. In an elegant experiment, Dr. Boulton's group compared patients provided with total contact casts with those provided with removable cast walkers. When the two groups were compared for duration of wound healing, those wearing removable cast walkers took significantly longer to heal due to removal of the casts when the people perceived themselves to be in a "safe" environment. This was elucidated using a waist-worn computerized accelerometer correlated with a second accelerometer hidden within the removable cast walker. Dr. Boulton called for a paradigm shift in the treatment of DFU and stressed that all future trials should have standardized offloading.

\section{Vascular biology and angiogenesis}

Old dogs learn new tricks- this title fitted well to a talk given by Drs. Klaus Preissner and Heidi Trussheim. Pointing out that cell-cell and cell-matrix interactions at sites of vascular injury determine the acute response of the vascular system to damage, some parallels to wound healing were identified. In the vascular context, the classical haemostatic factors are associated with; blood clotting, fibrinolysis, wound repair and vessel regeneration. However, as indicated by knockout mice and studies on patients with vascular diseases, these factors are important in vascular development and in processes related to vascular repair. One example, outlined in detail, was the role of platelets in pathological angiogenesis. It is long believed that platelets are major contributors to pathological angiogenesis because of their rich content in various agonists such as ADP, serotonin, PAF, von Willebrand factor etc. This hypothesis was tested in the mouse model of retinopathy of prematurity. Three different approaches to inhibit platelet function were tested in this model: (i) reduction of platelet numbers by antibody, (ii) inhibition of platelet adhesion, and (iii) inhibition of platelet function by aspirin. All approaches were associated with a 40-50 \% reduction in new vessel formation. In vitro assays in which endothelial cells were tested for the effect of platelet releasate revealed a synergistic promotion with bFGF on sprouting angiogenesis. Part of this effect was mediated by VEGF and lysolipids such as sphingosine-1phosphate, as supported by inhibition experiments. The likely consequences of the platelet-mediated cellcell interaction comprise endothelial disintegration with subsequent deposition of plasma components and activation and proliferation of endothelial cells. A second aspect related to the pathogenesis of atherosclerosis in diabetes was brought up, introduced by the reminder that multiple factors involved in haemostasis are present at the vascular wall at various stages of vessel degeneration. In search of molecules starting the process, it was found that lysates of smooth mus- 
cle cells were able to activate coagulation. It was subsequently found that extracellular RNA was the culprit, and that DNA had no effect. RNA was found to be a potent cofactor of contact phase activation, supported by binding experiments of RNA to components of the contact phase system. Thus RNA could be one long-sought initiation factor in blood clotting in particular, as the levels of circulating RNA are high in patients with vascular disease, and the pro-coagulant effect has a memory effect. It is thus speculated that RNA may serve as a novel thrombotic risk factor.

Dr. Christer Betsholtz, presenting on cell-cell interactions in angiogenesis, showed morphological data on unprecedented resolution in the developing retina as his model. Work together with Holger Gerhardt is based on the developmental aspects of the vessel system. The two models used to study the question of the sprouting tip in angiogenesis are the embryonic hindbrain and the postnatal developing retina of the mouse. In general, vessels develop by endothelial cell recruitment followed by pericyte recruitment. Vessels stabilize and specialize in a tissue-dependent context, giving rise, for example, to leaky vessels in the kidney and tight vessels in the retina. Studying the nature of the sprouting tip showed that the tip endothelial cell is not proliferating and that the tip has not yet a lumen, while the stalk cells have high proliferating capacities. Tip cells extend filopodia rich in actin filaments. Astrocyte-derived VEGF guides the VEGR-2 expressing filopodia via a gradient. Astrocytes are dependent on PDGF-A secreted from ganglion cells during the development of the retina. Note, VEGF secreted from astrocytes is not downregulated by hypoxia. Filopodia formation is a direct consequence of VEGF-A, as shown by ectopic expression experiments. Conversely, sequestering VEGF-A resulted in the disappearance of filopodia. Polarization of filopodia is strongly determined by sequestered VEGF, which is dependent on the heparin-binding properties of the specific isoforms. Studies also addressed the question of branching, which is a crucial step in angiogenesis. The „when and where" focussed on microglia which consistently appeared when turning and branching of vessels became apparent in the retina. Microglia are present both in the superficial and in the deep layers of the retina. These cells are essential to attract filopodia, but the factor involved may be distinct from VEGF-A. Studies in CSF-1 mutant mice, which lack microglial cells, indicated that filopodia formation was still possible in the absence of microglia, but they were less frequent. Finally, evidence was presented that the PDGF-B/receptor ß system plays a major role in vascular development and latency. In particular, pericytes have a protective role over endothelial cells with major effects on endothelial cell survival and permeability. Consistent with the previous hypothesis that pericytes inhibit endothelial cell proliferation, data were obtained from transgenic mice, showing that the elim- ination of pericytes by more than $50 \%$ using conditional endothelial-specific inactivation of PDGF-B induces proliferative retinopathy. From these results, it is clear that pericytes are important for the protection of endothelial cells, and for their proliferative capacity in vivo.

Dr. Roy Bicknell spoke about strategies to identify novel tissue specific genes. The endothelium is an excellent candidate as it expresses more mRNA than any other cell type. After constructing endothelial and non-endothelial cDNA libraries, sequences were extracted from dbEST using sequence retrieval systems. The pool of ESTs in the endothelial pool was found to be rather small, and almost 100\% accuracy was found when comparing these ESTs with SAGE libraries. From five known endothelial genes in the pools (vWF, VEGFR1 and 2, Tie-1 and -2), two had one hit each in the non-endothelial pool, but a total of 34 hits in the endothelial pool. Note, VEGR1 was not identified in either pool. Tie-1 tags also appeared in several nonendothelial SAGE libraries, in particular in tumour SAGE. RT-PCR control of five candidate genes selectively expressed in endothelial cell pools (ECM1-3, Robo 1, multimerin) proved correct. Subsequently, studies focussed on Robo 4, a member of the roundabout gene family, which have ligands called slit, and which are involved in axonal guidance. The primary structure includes two Ig-like domains, a fibronectintype domain and a conserved tail. Its expression is endothelial specific, it is not regulated by hypoxia or VEGF, and it is predominantly expressed in embryonic tissue and turned off after birth. There is evidence that Robo 4 is the only family member expressed in primary endothelial cells. It binds slit 2 , which inhibits endothelial cell migration. Although conflicting data related to the expression of Robo 1 and 4 in endothelial cells exist, it appears that Robo 4 has little effect on proliferation, but a strong effect on migration of endothelial cells. Robo 4 Fc strongly inhibits aortic ring sprouting. Thus, Robo expression is interesting with regard to its endothelial specificity, its selective expression in angiogenesis and its possible therapeutic application.

Dr. Jennifer LeCouter gave an insight into the biology of VEGF and other regulators of angiogenesis. This keynote lecture addressed three important facets of VEGF biology: its role in physiological and pathological angiogenesis, VEGF receptor biology, and new mediators of angiogenesis related to VEGF. It is emphasized that VEGF plays a central role in developmental angiogenesis, and that VEGF acts strictly in a dose-dependent manner. Among the increasing list of properties, VEGF is a survival factor for postnatal EC, and is critical for bone development and physiological angiogenesis in the ovary. VEGF has been associated with malignancy, and blockade of VEGF by antibody technologies is the first proof of the principle that an anti-angiogenic strategy in addition to standard che- 
motherapy is an effective strategy to treat human cancer. From studies in transgenic mice, it has evolved that the VEGF receptors 1 (flt) and 2 (flk) have essential but distinct functions during development. While R2 appears to mediate most of the known functions of VEGF, R1 acts as a decoy receptor preventing VEGF from activating R2. However, via its function of mononuclear cells, activation of R1 can also affect angiogenesis via recruitment of monocytes. Using receptor-specific agonists, activation of ERK1/2 and p38 were found upon stimulation of R2. Using liver cells as a model, a distinct paracrine function of VEGF and a new role of the dual R1/R2 system was identified. Stimulation of R1 in liver sinusoidal EC induced the production of cytokines such as HGF and hepatocyte proliferation, while stimulation of R2 induced liver sinusoid endothelial proliferation. Thus, VEGF acts on liver sinusoidal endothelial cells (LSEC) via R2 to induce cell proliferation, while its function via R1 is cytokine production for hepatocyte growth. This finding also emphasizes that VEGFs paracrine signals are tissue specific, and that tissue protection can be uncoupled from angiogenesis. Other tissue-specific VEGFlike molecules may exist, of which EG-VEGF is one paradigm. It is not a member of the VEGF family, and gives signals via G-coupled receptors inducing MAPK via the EG-VEGFR1, and inducing PI-3-Kinase, Akt and eNOS via EG-VEGFR2. EG-VEGF does not induce angiogenesis in the eye, as its expression is restricted to steroidogenic tissues. As such, it is highly expressed in ovarian stroma of patients with polycystic ovary syndrome, making it a suitable therapeutic target. Thus, angiogenesis is a highly tissue-specific and complex process. The diversity of responses to VEGF and its inhibition is determined by the vascular-bed-specific signalling and the tissue-specific regulators in a given situation.

Dr. Gavin Thurston gave a talk on the angiopoietins and their role in vascular biology. He emphasized the cell-specific dual effects of the angiopoietins $1 \& 2$, and reminded the audience that the function of Tie-1 is still not known. Ang-1 deficiency impairs the interaction between endothelial cells and pericytes/SMC, and Ang-1 administration to the developing retina affects proper pericyte recruitment, while Ang-1 and VEGF determine the plasticity window during these early time points. Later, Ang-1 loses its effect on vessel plasticity. However, Ang-1 can be used to inhibit pathological vessel permeability (such as in diabetic retinopathy), either from inflammatory or from VEGF signalling. Ang-1 reduces VEGF and ICAM-1 expression in acute models of hyperglycaemia, and it reduces leukocyte adhesion and endothelial cell damage in diabetic mice. However, Ang-1 fails to inhibit inflammatory signals in chronic models or in models associated with strong infiltrating cell components. Ang-2, the context-specific antagonist of Ang-1, has potent vascular remodelling properties, according to published studies using mice with a LacZ knock-in construct. These mice show major defects in sprouting angiogenesis in the retina, while the hyaloid vessels persist for compensatory perfusion in the peripheral retina. Most interestingly, these mice, which exhibit defects in lymphatic vessel formation, show that the VEGF and angiopoietin family have complementary roles in angiogenesis and lymphangiogenesis. Lastly, the new high throughout approach „Velocigene" was presented which allows the circumvention of the usual bottleneck of transgenic and knockout technologies. With this technologies, a large list of genetic deletions related to angiogenesis were made, or are in the process of being made, and reveal that the family of angiopoietin relatives is also involved in specific processes of metabolism.

\section{Diabetic retinopathy}

In a series of experiments, Drs. Maria Grant and Aqeela Afzal discussed the important question of whether, and to what extent haematopoietic stem cells can contribute to vessel repair and angiogenesis, with particular emphasis on the retina. Prior studies had shown that adult stem cells are able to contribute to organ repair and formation. Haematopoietic stem cells can be mobilized from adult bone marrow, and contribute to angiogenesis at ischaemia sites. They can complement neo-angiogenesis that derives from resident endothelium. The basic concept is that the ischaemic site gives signals via hypoxia-inducible growth factors such as VEGF to the bone marrow, inducing proteolytic activity in the bone marrow. Using bone marrow from GFP+ mice, and laser-induced retinal neovascularization as a model, it was shown in a series of experiments that adult haemangioblasts are recruited to active sites of neovascularization, and become incorporated into the newly formed vessel, which eventually opens for blood to flow. A mechanism required for such cellular transition includes the opening of the blood retinal barrier. A candidate molecule is stromal-derived factor 1 alpha, a chemokine that regulates cell trafficking. It is up-regulated in inflammation and may be involved in diabetic macula oedema and angiogenesis. Preliminary experiments using anti-SDF strategies found reduced subretinal neovascularization in a model of laser-induced choroidal neovascularization, and reduced retinal permeability, suggesting that modulation of SDF-1alpha may prevent retinal and subretinal angiogenesis and macula oedema formation.

Gene Profiling of Diabetic Complications of the Retina was the title of the talk given by Drs. Anand Swaroop and Monica Buraczynska. Advanced stages of diabetic retinopathy and age-related macular degeneration have similarities, as both are major causes of untreatable blindness, are complex and multifactorial 
in nature, show balanced interactions between environmental and genetic factors, and have oxidative damage in their list of pathogenic factors. While ongoing studies are trying to identify susceptibility and modifier genes for both diseases without definitive results yet, the long-term goal is to identify the mechanisms underlying the adaptive response of the retina to chronic hyperglycaemia. To this end, data are preliminary but include comprehensive gene profiling of retinae from diabetic mice treated with various drugs, and from mice with genetic modifications. In collaboration with Tim Kern, Cleveland, they chose different time points in relation to well-defined functional and structural changes in the STZ-diabetic rat model to study gene arrays in the eye. At two months of diabetes, most of the differentially regulated genes in diabetes were found downregulated more than two-fold, the majority belonging to the crystallin family. Confirmation experiments revealed expression of almost all crystallins also in the retina, possibly associated with their functions as chaperons. Aspirin, but not aminoguanidine or vitamin $\mathrm{E}$ normalized the downregulated expression of most of the crystallins examined. Further work addresses other differentially expressed genes.

Novel proteomics techniques for use in diabetes research are now on the horizon. Invited speaker, Dr. Peter James gave an overview on developing high throughput protein analysis for population-based proteomics. The diversity of an individual, although imprinted in its genes, is best reflected by its proteins, whose numbers approximate 1 million. The correlation between RNA and proteins is weak, in particular in secreted proteins. The platform on which important information related to diseases such as cancer, diabetes and vascular diseases, are gathered is broadening: rapidly increasing sequence information, mRNA expression data, protein expression data, metabolomic data, and population and SNP data become available through high throughput methods. Proteomic modes of analysis include 2D gel electrophoresis, non-gel chromatography methods (ICAT, MCAT, PIQS), and chip/strip technologies, all of which have specific advantages and disadvantages related to preparative efforts, reflection of the dynamic range, feasibility of pattern matching etc. With these techniques, and the plasma as the starting material, one approach to analyze an individual protein pattern is fluorescent difference gel electrophoresis. The mass spectrometric analysis of the tryptic protein or peptide fragments yields fingerprints, which allow comparison with established expression pattern. As this method is costly, alternatives are sought, one of which may be chip analysis of proteins based on antibody binding. However, despite the major potential of this technique, a variety of unsolved problems are associated with it, including specificity of antibodies, antibody production, chip logistics, recognition of denatured proteins etc. Recently, a new method was proposed, which uses frozen tissue sections as starting material. A mass spectrum is obtained by laser scan, which allows molecular imaging of tissues down to single cell level. The medium-term commitment evolving from current research and progress in this field is to generate a marker set of approximately 100 proteins based on their predictive value for a specific disease, and to generate a chip for which testing is feasible within around 15 minutes at low cost.

\section{Diabetic nephropathy}

One session focussed on recent advances in our understanding of diabetic renal disease with a major emphasis on new aspects of the cellular and molecular changes that are postulated to play pivotal roles in the development and progression of this disorder.

Dr. Sale and Dr. Rich from the Wake Forest University School of Medicine, addressed the genetic factors that may play a role in susceptibility to this complication. Based on studies in mono and in dizygotic twins, as well as in other family studies using a sib pair design approach, it has been shown that, although genetic factors predominate environmental factors also play a role. Heritable factors are implicated for both functional (e.g. albumin/creatinine ratio) and structural (e.g. glomerular and tubular basement membrane thickness and fractional mesangial volume) manifestations of the disease. Both candidate geneand genome-wide scanning approaches are currently being used, and these are being applied to both Type I and Type II diabetes. Various candidate genes such as matrix metalloproteinases (MMPs) and interleukin 1 alpha have been identified, and using genome-wide scanning strong linkage signals, have been noted on chromosomes 7, 12 and 18 .

Drs. Viberti and Setti from Guys Hospital, London addressed the interactions between metabolic and haemodynamic insults in mediating glomerulosclerosis. Natural history studies of Type I diabetic patients have identified roles for both systemic blood pressure and glycaemic control in influencing the progression of diabetic nephropathy. A range of cell culture studies has shown that stretch, an in vitro counterpart of systemic hypertension, induced expression of a range of growth factors, chemokines and extracellular matrix proteins. Furthermore, angiotensin II acts in a synergistic manner with stretch to promote expression of VEGF. This phenomenon is possibly partly explained by the ability of stretch to up-regulate expression of the angiotensin type I receptor subtype. Further in vitro studies in human mesangial cells have identified a role for IGF-1 via a src-dependent pathway, in inducing VEGF production. Recent studies by this group have shown that the glucose transporter glut-1 is activated by haemodynamic perturbations such as stretch. 
This promotes glucose uptake into mesangial cells with subsequent activation of intracellular signalling molecules, as well as the hexosamine pathway by increasing the enzyme GFAT ultimately leading to increased growth factor expression and extracellular matrix accumulation. Intracellular signalling molecules such as protein kinase $\mathrm{C}$ (PKC) and p38 MAP kinase also appear to play a central role in mediating the effects of both haemodynamic and metabolic insults. The importance of intracellular signalling pathways involving PKC and p38 MAP kinase in mediating the injurious effects of metabolic and haemodynamic insults continues to be unravelled by these and other investigators and is a major component of ongoing research in this field. These in vitro studies now need to be translated to the in vivo context.

Dr. Herman Haller and Dr. Matthias Meier extended this issue of intracellular signalling molecules by focussing on PKC in diabetic nephropathy. After summarising the current state of knowledge of the various $\mathrm{PKC}$ isoforms, important changes in one specific isoform, PKC alpha, were described. This isoform is translocated by glucose and antisense approaches have been used to further define the role of this intracellular signalling molecule. Exciting preliminary data obtained from diabetes-induced PKC alpha knockout mice were consistent with $\mathrm{PKC}$ alpha playing a major role in the development of albuminuria possibly via a VEGF-dependent pathway in diabetes. Furthermore, there were changes in expression of the heparan sulphate proteoglycan, perlecan, that plays a role in albumin permeability. Further studies by this group and others are continuing to explore the role of PKC in diabetic complications, the relative importance of the various isoforms remaining an area of ongoing controversy and investigation.

Dr. Kumar Sharma and Dr. Satish Rao described recent findings linking the prosclerotic growth factor, transforming growth factor $\beta$ (TGF $\beta$ ), to progressive renal injury in diabetes. Recent data from his own and other groups were described, in which potential additive renoprotective effects of blocking TGF $\beta$ in the setting of concomitant ACE inhibitor treatment were identified. The molecular mechanisms responsible for the stimulation of extracellular matrix proteins by TGF $\beta$ are increasingly being clarified. For example, TGF $\beta$-induced type I collagen expression is totally SMAD 4-dependent whereas fibronectin expression appears to be independent of SMAD 4. Further effects of TGF $\beta$ were described, which may be relevant to diabetes including effects of this growth factor on inositol phosphate $3\left(\mathrm{IP}_{3}\right)$ receptors and sensitivity to $\mathrm{IP}_{3}$. Although most studies on TGF $\beta$ in diabetes have examined the $\beta 1$ isoform, the $\beta 2$ isoform also appears to be important and is up-regulated in diabetes. The importance of TGF $\beta$ is currently under examination using a range of approaches including neutralising antibodies, soluble receptors to the TGF $\beta$ type II receptor, kinase inhibitors and SMAD inhibitors. In addition, antifibrotic drugs such as pirfenidone are currently in clinical trial to specifically address whether antisclerotic strategies have an important renoprotective role in diabetes.

Drs. Stuart Shankland and Raghu Durvasala reviewed the importance of cell cycle regulatory proteins in diabetic nephropathy. It has been postulated that the structural hallmarks of this disease, including renal hypertrophy, loss of glomerular epithelial cells and accumulation of extracellular matrix, may partly occur as a result of altered expression of proteins implicated in the cell cycle, including regulatory proteins (e.g. CDK4, CDK2) and inhibitors known as cyclin kinase inhibitors (e.g. p21, p27). In the diabetic mouse, various cyclin kinase inhibitors (CKI), including p21 and p27 have been reported to be increased. Furthermore, animals with gene deletions of either p21 or p27, are resistant to the injurious effects of diabetes on renal structure. These CKIs appear to play a pivotal role in mediating renal hypertrophy, a prominent feature of the diabetic kidney. Preliminary studies where more than one CKI has been deleted provide further evidence linking these proteins to diabetes-associated glomerular hypertrophy. Further studies by this group have been examining the mechanisms responsible for the loss of the glomerular epithelial cell, the podocyte, in diabetes. This event appears to be closely linked to the pathogenesis of proteinuria in diabetes but the exact mechanism or mechanisms responsible for podocyte loss are as yet not fully determined. Possible explanations for this phenomenon including the role of apoptosis, lack of proliferation and detachment of these cells, were discussed and are areas of ongoing research by many groups.

Dr. Erwin Bottinger and Dr. Katalin Shustak reviewed recent data that have been obtained in collaboration with Dr. Sharma at Thomas Jefferson University, as part of an NIDDK, NHLBI and JDRF initiative to explore and develop animal models of diabetic renal disease (AMDCC - www.amdcc.org). This group has been using high-throughput microarray analyses to identify genes linked to experimental diabetic nephropathy. These studies have been performed in the setting of concomitant experiments, which involve detailed functional and structural assessment of the various models of diabetic renal disease. Most work is being performed in mice, both streptozotocin-induced and genetically diabetic $(\mathrm{db} / \mathrm{db})$ mice. Three specific genes that appeared to be modulated in the diabetic kidney were discussed in detail. Firstly, mRNA levels for a specific enzyme were reported to be reduced, the functional significance of the decrease in this enzyme is as yet not fully evident. Osteopontin gene expression was reported to be altered in the diabetic kidney and further immunohistochemical studies are in progress to confirm these changes at the protein level. Finally, CD36 was noted to be markedly up-regulated in the diabetic 
kidney. This protein is involved in transport of fatty acids and is a multiligand scavenger receptor. Ligands include oxidised LDL and various glycated proteins. CD36 is found on many cells including macrophages, platelets and endothelial cells. Its function in the kidney is unknown but it is localised to tubular cells, can mediate apoptotic signals and possible activates p38 MAP kinase. Dr. Bottinger emphasised both the strengths and pitfalls of microarray analysis, reviewed the advantages and disadvantages of Affymetrix software and indicated that much of the future work in this area in a heterogenous organ such as the kidney will require more novel ways to separate glomeruli and tubules for subsequent detailed molecular analysis.

\section{Round-up of potential therapeutic strategies:}

From these talks emerged a clear translational message: There are a number of potential therapeutic approaches that can be taken to intervene at different points along the pathway towards end-organ damage. These strategies to prevent or reverse diabetic complications were briefly discussed at the end of the workshop and are outlined below.

The first strategic aim should be to attempt to 'stop the engine' by the careful control of hyperglycaemia. The effectiveness of this first approach has been demonstrated by results of the DCCT/EDIC cohort, which clearly demonstrated that maintaining tight glycaemic control through intensive insulin therapy reduces the overall incidence of microvascular complications.

Following this, early treatment strategies that aim to treat before excessive microvascular pathology develops need to be deployed. Here, the use of effective agents targeting the main mechanisms of intracellular injury needs to be fully investigated. Such strategies include investigating existing clinically available compounds that have demonstrated efficacy in the laboratory (e.g. the synthetic thiamine derivative Benfotiamine) to block damage mediated by activation of transketolase (TK) and antioxidants such as $\alpha$-lipoic acid which can prevent or reverse deficits in blood flow and nerve conduction. In addition there are a number of compounds currently in commercial development that may prove efficacious at this point. These include but are not limited to: PARP inhibitors, crosslink breakers and small molecule antagonists of RAGE and SOD mimetics.

Finally, a cohesive therapeutic strategy might employ a 'cluster bomb' approach using multiple, synergistically acting therapeutics with multisite actions that target microvessels and specific cells within target end-organs to prevent the devastating complications that occur through diabetes.

Acknowledgements: The Oxford Workshops are hosted and organized jointly by EASD and the Juvenile Diabetes Research Foundation. The scientific Chairs for this 8th Workshop were Drs. Mark Cooper and Hans-Peter Hammes and the JDRF scientific representative was Dr. Antony Horton. The Workshop series scientific coordinator is Dr. Philippe Halban. EASD and JDRF thank Mrs. Mary Hata (EASD) and Ms. Caroline Wood (local organiser) for their help in organising this meeting and the Aventis Company for their most generous support.

\section{This report was written by:}

\section{Mark Cooper}

Baker Heart Research Institute

Melbourne, Australia

Hans-Peter Hammes

University of Heidelberg

Germany

Antony Horton

JDRF

New York 


\title{
European Foundation for the Study of Diabetes
}

The Research Foundation of the European Association for the Study of Diabetes

\section{Report on an EFSD/MSD Travel Fellowship for Young Scientists 2003}

\author{
Marieke B. Snijder \\ Institute for Research and Extramural Medicine, VU University Medical Center, \\ Amsterdam, The Netherlands
}

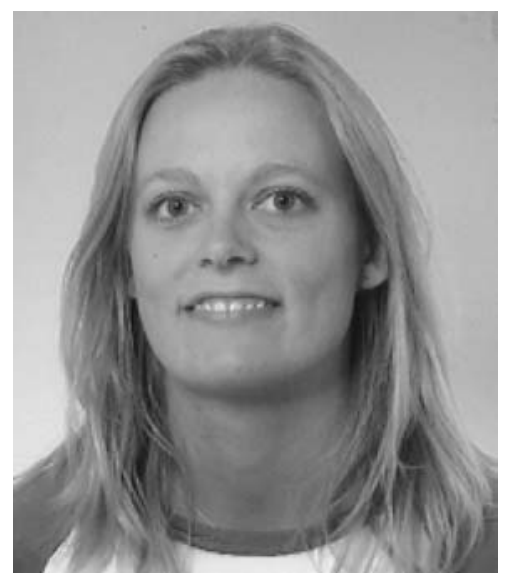

I would like to thank the European Foundation for the EFSD/MSD Travel Fellowship for Young Scientists, which enabled me to visit the International Diabetes Institute in Melbourne, Australia, between January and April 2003. The application was submitted in the context of my PhD project entitled 'Body composition in relation to Type 2 diabetes and cardiovascular disease risk. The role of different fat depots and lean tissue'.

It is generally known that obesity is associated with a higher risk of Type 2 diabetes, and particularly abdominal obesity (often measured by waist-to-hip ratio) has been shown to be an important predictor of insulin resistance and Type 2 diabetes. It is generally thought that the waist-to-hip ratio (WHR) reflects the amount of visceral fat causing the metabolic disturbances. Waist circumference is the strongest correlate of visceral fat. Waist circumference, however, is not always a stronger predictor of Type 2 diabetes and other cardiovascular risk than is WHR. A higher WHR can be the result of a larger waist as well as smaller hips. This suggests that smaller hips (representing either lower muscle mass and/or lower fat mass at the hips) may be involved in determining the increased risk associated with an increased WHR. We investigated the contribution of hip circumference and found that independent of a large waist circumference and overall obesity (BMI), a larger hip circumference was associated with lower glucose levels and lower risk of Type 2 diabetes (Snijder et al., 2003, Obes Res; Snijder et al., 2003, Am J Clin Nutr). Mechanisms to explain these associations are not entirely clear yet.

This interesting relation has been investigated in only a few studies, which did not use an oral glucose tolerance test (OGTT) to define diabetes and were usually performed in elderly subjects only. It is important to see whether these associations can be confirmed in other large study populations including all ages and with a proper definition of diabetes. Furthermore, these studies have been performed only in Caucasian populations. Because there are large differences in body composition between races, the question is whether these associations found in Caucasians also exist in other ethnic groups.

Prof. Zimmet and Dr. Shaw of the International Diabetes Institute in Melbourne have great expertise in the field of Type 2 diabetes. Under their supervision, the Australian Diabetes, Obesity and Lifestyle Study (AusDiab Study) was conducted. This is a large, cross-sectional, population-based survey of diabetes prevalence and associated risk factors. Also data from Mauritius, Rodrigues, Nauru, and Papua New Guinea are available at the institute, which enabled me to study different races (Micronesians, Melanesians, Indians and Creoles). In all their studies, glucose tolerance status is measured by OGTT, and an extensive physical examination was performed, including waist and hip circumferences. These studies formed an excellent opportunity for me to answer some important questions about the relation between body composition and Type 2 diabetes.

In the AusDiab Study, we confirmed the cross-sectional results of the Hoorn Study by showing that a larger hip circumference was independently associated with lower glucose levels, after adjustment for waist circumference, BMI, and age. Furthermore, we extended our observations by considering additional components of the metabolic syndrome as outcome, i.e. hypertension and dyslipidemia. The AusDiab Study covers a wide age range (all ages above 25 years), and the independent association of larger hip circumference with more favourable levels of glucose, triglycerides and HDL-cholesterol, was apparent across all age groups, except in persons aged 75 years or older. This work resulted in a paper which was recently published (Snijder et al., 2004, Int J Obes). 
Results on the relation between body composition and components of the metabolic syndrome in the population studies of Mauritius, Rodrigues, Nauru, and Papua New Guinea have not been published yet.

This project contributed to the field of body composition, Type 2 diabetes, and the metabolic syndrome and has already stimulated later work carried out at the Institute for Research in Extramural Medicine in Amsterdam. For example, we have shown that accumulation of fat mass in the legs as well as a larger lean (muscle) mass in the legs (measured by dual energy $\mathrm{x}$-ray absorptiometry) was associated with a better glucose tolerance status, after adjustment for trunk fat mass (Snijder et al., 2004, Dia-

\section{Announcements}

\section{International Colloquium on Diabetes and Pregnancy}

Chennai, India, 11-12 December 2004

For further information please contact:

Dr Madhuri S Balaji

Tel: +91 4426412296 / 26615757 / 20015050

Fax: +91 4428295467

E-mail:vseshiah@ hotmail.com,vseshiah@eth.net Website: http://seshiahinstitute.com/gdmconference betes Care). It is hypothesised that, due to lower lipolytic activity compared to abdominal fat, femoralgluteal fat is more likely to take up free fatty acids (FFA) from the circulation. Hereby, it protects other organs against high FFA exposure, which is associated insulin resistance. Also differences between abdominal and femoral-gluteal fat depots in secretion of adipokines, which are involved in the glucose metabolism, may be involved in the pathophysiological mechanism. More pathophysiological research, however, is needed.

I am grateful to Prof. Zimmet and Dr. Shaw for welcoming me to their institute and for making my visit very worthwhile.
International Endovascular Laparoscopic Congress Québec City Convention Center, 20-22 May 2004

For further information please check the following website:

http://www.endovascularlaparoscopy.org 